Journal of Universal Mathematics

Vol.2 No.2 PP.113-126 (2019)

ISSN-2618-5660

\title{
ON THE CAUCHY PROBLEM FOR MATRIX FACTORIZATIONS OF THE HELMHOLTZ EQUATION
}

\author{
D.A. JURAEV
}

\begin{abstract}
In the paper it is considered the regularization of the Cauchy problem for systems of elliptic type equations of the first order with constant coefficients factorisable Helmholtz operator in two-dimensional unbounded domain. Using the results of the works [20], [21], [22], [23], [24], [25] and [26], we construct in explicit form Carleman matrix and based on the regularized solution of the Cauchy problem.
\end{abstract}

\section{INTRODUCTION}

This problem concerns ill-posed problems, i.e. it is unstable. It is known that the Cauchy problem for elliptic equations is unstable relatively small change in the data, i.e. incorrect (example Hadamard, see, for instance [10], p. 39). There is a sizable literature on the subject (see, e.g. [4]-[9], [13]). Tarkhanov [2] has published a criterion for the solvability of a larger class of boundary value problems for elliptic systems. In unstable problems, the image of the operator is not is closed, therefore, the solvability condition can not be is written in terms of continuous linear functionals. So, in the Cauchy problem for elliptic equations with data on part of the boundary of the domain the solution is usually unique, the problem is solvable for everywhere dense a set of data, but this set is not closed. Consequently, the theory of solvability of such problems is much more difficult and deeper than theory of solvability of Fredholm equations. The first results in this direction appeared only in the mid-1980s in the works of L.A. Aizenberg, A.M. Kytmanov, N.N. Tarkhanov (see, for instance [3]).

The uniqueness of the solution follows from Holmgren's general theorem (see [14]). The conditional stability of the problem follows from the work of A.N. Tikhonov (see [13]), if we restrict the class of possible solutions to a compactum.

In this paper we construct a family of vector-functions $U_{\sigma(\delta)}(x)=U\left(x, f_{\delta}\right)$ depending on a parameter $\sigma$, and prove that under certain conditions and a special choice of the parameter $\sigma=\sigma(\delta)$, at $\delta \rightarrow 0$, the family $U_{\sigma(\delta)}(x)$ converges in the usual sense to a solution $U(x)$ at a point $x \in G$.

2000 Mathematics Subject Classification. Primary 35J46; Secondary 35J56.

Key words and phrases. The Cauchy problem, Regularization, Factorization, Regular solution, Fundamental solution.

The author was supported in part by Grant \#OT-F-4-03, "Concrete dynamic systems with continuous and discrete time, spectra of partially integral operators" (2017-2021) of Karshi State University. 
Following A.N. Tikhonov (see [13]), a family of vector-valued functions $U_{\sigma(\delta)}(x)$ is called a regularized solution of the problem. A regularized solution determines a stable method of approximate solution of the problem. For special domains, the problem of extending bounded analytic functions in the case when the data are given only on a part of the boundary was considered by Carleman (see [4]). The researches of T. Carleman were continued by G.M. Goluzin and V.I. Krylov (see [12]). A multidimensional analogue of Carleman's formula for analytic functions of several variables was constructed in (see [11]). The use of the classical Green's formula for constructing a regularized solution of the Cauchy problem for the Laplace equation was proposed by Academician M.M. Lavrent'ev (see, for instance [5], [6]). Using the ideas of M.M. Lavrent'ev and Sh. Yarmukhamedov, a regularized solution of the Cauchy problem for the Laplace and Helmholtz equations was constructed in explicit form (see, for instance [7], [8], [9]). In [1] an integral formula is proved for systems of equations of elliptic type of the first order, with constant coefficients in a bounded domain. In [16], the Cauchy problem for the Helmholtz equation in an arbitrary bounded plane domain with Cauchy data, known only on the region boundary, is considered. The solvability criterion for the Cauchy problem for the Laplace equation in the space $\mathbb{R}^{m}$ it was considered by Shlapunov in work [17].

For systems of equations of elliptic type of the first order with constant coefficients, the factorizing operator of Helmholtz, in [22] the validity of the integral formula in a three-dimensional unbounded domain was proved.

The construction of the Carleman matrix for elliptic systems was carried out by: Sh. Yarmukhamedov, N.N. Tarkhanov, A.A. Shlapunov, I.E. Niyozov, D.A. Juraev and others. The system considered in this paper was introduced by N.N. Tarkhanov. For this system, he studied correct boundary value problems and found an analogue of the Cauchy integral formula in a bounded domain.

The system considered in this paper was introduced by N.N. Tarkhanov. For this system, he studied correct boundary value problems and found an analogue of the Cauchy integral formula in a bounded domain (see, for instance [3]).

In many well-posed problems for systems of equations of elliptic type of the first order with constant coefficients that factorize the Helmholtz operator, it is not possible to calculate the values of the vector function on the entire boundary. Therefore, the problem of reconstructing the solution of systems of equations of first order elliptic type with constant coefficients, factorizing the Helmholtz operator (see, for instance [20], [21], [22], [23], [24], [25] and [26]), is one of the topical problems in the theory of differential equations.

For the last decades, interest in classical ill-posed problems of mathematical physics has remained. This direction in the study of the properties of solutions of the Cauchy problem for the Laplace equation was started in [5]-[8], [10] and subsequently developed in [1]-[3], [17]-[26].

In this paper, we present an explicit formula for the approximate solution of the Cauchy problem for the matrix factorizations of the Helmholtz equation in a bounded region on the plane. The two-dimensional case requires special consideration, in contrast to three or more dimensions in many mathematical problems. Our formula for an approximate solution also includes the construction of a family of fundamental solutions for the Helmholtz operator on the plane. This family is parametrized by some entire function $K(w)$, the choice of which depends on the dimension of the space. This motivates a separate study of regularization formulas 
in flat domains and leads to improved estimates compared to the three-dimensional case.

\section{The INTEGRAL FORMUla IN AN UNBOUNDED DOMAIN}

Let $\mathbb{R}^{2}$ be the two-dimensional real Euclidean space,

$$
x=\left(x_{1}, x_{2}\right) \in \mathbb{R}^{2}, y=\left(y_{1}, y_{2}\right) \in \mathbb{R}^{2} .
$$

$G \subset \mathbb{R}^{2}$ be an unbounded simply-connected domain with piecewise smooth boundary consisting of the plane $T: y_{2}=0$ and some smooth curve $S$ lying in the half-space $y_{2}>0$, i.e., $\partial G=S \bigcup T$.

We introduce the following notation:

$$
\begin{gathered}
r=|y-x|, \alpha=\left|y_{1}-x_{1}\right|, w=i \sqrt{u^{2}+\alpha^{2}}+y_{2}, u \geq 0, \\
\frac{\partial}{\partial x}=\left(\frac{\partial}{\partial x_{1}}, \frac{\partial}{\partial x_{2}}\right)^{T}, \frac{\partial}{\partial x} \rightarrow \xi^{T}, \xi^{T}=\left(\begin{array}{c}
\xi_{1} \\
\xi_{2}
\end{array}\right) \text { be a transposed vector } \xi \\
U(x)=\left(U_{1}(x), \ldots, U_{n}(x)\right)^{T}, u^{0}=(1, \ldots, 1) \in \mathbb{R}^{n}, n=2^{m}, m=2, \\
E(z)=\left\|\begin{array}{c}
z_{1} \ldots 0 \\
\ldots \ldots \\
0 \ldots z_{n}
\end{array}\right\| \text { be a diagonal matrix, } z=\left(z_{1}, \ldots, z_{n}\right) \in \mathbb{R}^{n} .
\end{gathered}
$$

Let $D\left(\xi^{T}\right),(n \times n)$ - dimensional matrix with elements consisting of a set of linear functions with constant coefficients of the complex plane for which the following condition is satisfied:

$$
D^{*}\left(\xi^{T}\right) D\left(\xi^{T}\right)=E\left(\left(|\xi|^{2}+\lambda^{2}\right) u^{0}\right),
$$

where $D^{*}\left(\xi^{T}\right)$ is the Hermitian conjugate matrix $D\left(x^{T}\right), \lambda$ is a real number.

We consider in the region $G$ a system of differential equations

$$
D\left(\frac{\partial}{\partial x}\right) U(x)=0
$$

where $D\left(\frac{\partial}{\partial x}\right)$ is the matrix of first-order differential operators.

We denote by $A(G)$ the class of vector functions in a domain $G$ continuous on $\bar{G}=G \bigcup \partial G$ and satisfying system $(2.1)$.

If $G$ is a bounded and $U(y) \in A(G)$, then the following integral formula of Cauchy type is valid (see [22])

$$
U(x)=\int_{\partial G} M(y, x) U(y) d s_{y}, \quad x \in G,
$$

where

$$
M(y, x)=\left(E\left(-\frac{i}{4} H_{0}^{(1)}(\lambda r) u^{0}\right) D^{*}\left(\frac{\partial}{\partial y}\right)\right) D\left(t^{T}\right) .
$$

Here $t=\left(t_{1}, t_{2}\right)$ is the unit external normal, drawn at a point $y$, the curve $\partial G$, $-\frac{i}{4} H_{0}^{(1)}(\lambda r)$ is the fundamental solution of the Helmholtz equation in $\mathbb{R}^{2}$. [15].

We denote by $K(w)$ is an entire function taking real values for real $w,(w=$ $u+i v, u, v$-real numbers) and satisfying the following conditions:

$$
K(u) \neq 0, \sup _{v \geq 1}\left|v^{p} K^{(p)}(w)\right|=\mathrm{M}(u, p)<\infty, \quad-\infty<u<\infty, p=0,1,2 .
$$


We define a function $\Phi(y, x)$ at $y \neq x$ for the following equation:

$$
\Phi(y, x)=-\frac{1}{2 \pi K\left(x_{2}\right)} \int_{0}^{\infty} \operatorname{Im} \frac{K(w)}{w-x_{2}} \frac{u I_{0}(\lambda u)}{\sqrt{u^{2}+\alpha^{2}}} d u .
$$

Here $I_{0}(\lambda u)=J_{0}(i \lambda u)$ is the Bessel function of the first kind is of zero order [14].

Formula (2.2) is true if instead $-\frac{i}{4} H_{0}^{(1)}(\lambda r)$ of substituting the function

$$
\Phi(y, x)=-\frac{i}{4} H_{0}^{(1)}(\lambda r)+g(y, x)
$$

where $g(y, x)$ is the regular solution of the Helmholtz equation with respect to the variable $y$, including the point $y=x$.

Then formula (2.2) has the following form

$$
U(x)=\int_{\partial G} M(y, x) U(y) d s_{y}, \quad x \in G,
$$

where

$$
M(y, x)=\left(E\left(\Phi(y, x) u^{0}\right) D^{*}\left(\frac{\partial}{\partial y}\right)\right) D\left(t^{T}\right) .
$$

Formula (2.6) is generalized for the case when $G$ is the unbounded domain.

Let $G \subset \mathbb{R}^{2}$ be an unbounded domain, with a piecewise smooth boundary $\partial G$ ( $\partial G$-extends to infinity).

We denote by $G_{R}$ the part $G$ lying inside the circle of radius $R$ with center at zero:

$$
G_{R}=\{y: y \in G,|y|<R\}, G_{R}^{\infty}=G \backslash G_{R}, R>0 .
$$

Theorem 2.1. Let $U(y) \in A(G), G$ be a finitely connected unbounded domain in $\mathbb{R}^{2}$, with piecewise-smooth boundary $\partial G$. If for each fixed $x \in G$ we have the equality

$$
\lim _{R \rightarrow \infty} \int_{G_{R}^{\infty}} M(y, x) U(y) d s_{y}=0
$$

then the formula (2.6) is true.

Proof. Indeed, for a fixed $x \in G(|x|<R)$ and taking (2.6) into account, we have

$$
\begin{gathered}
\int_{\partial G} M(y, x) U(y) d s_{y}=\int_{\partial G_{R}} M(y, x) U(y) d s_{y}+ \\
+\int_{\partial G_{R}^{\infty}} M(y, x) U(y) d s_{y}=U(x)+\int_{\partial G_{R}^{\infty}} M(y, x) U(y) d s_{y}, x \in G_{R} .
\end{gathered}
$$

Taking into account condition (2.7), for $R \rightarrow \infty$, we obtain (2.6).

Suppose $G$ that an unbounded domain lies inside a strip of the smallest width defined by inequality

$$
0<y_{2}<h, h=\frac{\pi}{\rho}, \rho>0,
$$

and $\partial G$ extends to infinity. 
Suppose that for some $b_{0}>0$ the length $\partial G$ satisfies the growth condition

$$
\int_{\partial G} \exp \left[-b_{0} \rho_{0}\left|y_{1}\right|\right] d s_{y}<\infty, 0<\rho_{0}<\rho .
$$

Suppose $U(y) \in A(G)$ that it satisfies the boundary growth condition

$$
|U(y)| \leq \exp \left[\exp \rho_{2}\left|y_{1}\right|\right], \rho_{2}<\rho, y \in G \text {. }
$$

In (2.4) we put

$$
\begin{gathered}
K(w)=\exp \left[-b i \rho_{1}\left(w-\frac{h}{2}\right)-b_{1} i \rho_{0}\left(w-\frac{h}{2}\right)\right], \\
K\left(x_{2}\right)=\exp \left[b \cos \rho_{1}\left(x_{2}-\frac{h}{2}\right)+b_{1} \cos i \rho_{0}\left(x_{2}-\frac{h}{2}\right)\right], \\
0<\rho_{1}<\rho, 0<x_{2}<h,
\end{gathered}
$$

where

$$
b=2 a \exp \left(\rho_{1}\left|x_{1}\right|\right), b_{1}>\frac{b_{0}}{\cos \left(\rho_{0} \frac{h}{2}\right)}, a \geq 0, b>0
$$

Then the integral representation (2.6) is true.

For a fixed $x \in G$ and $y \rightarrow \infty$, we estimate the function $\Phi(y, x)$ and its derivatives $\frac{\partial \Phi(y, x)}{\partial y_{j}}, j=1,2$. For the estimation $\frac{\partial \Phi(y, x)}{\partial y_{j}}$ we use equalities

$$
\begin{gathered}
-2 \pi K\left(x_{2}\right) \frac{\partial \Phi(y, x)}{\partial y_{1}}=\frac{\left(y_{1}-x_{1}\right) \operatorname{Re} K\left(w_{0}\right)-\operatorname{sign}\left(y_{1}-x_{1}\right)\left(y_{2}-x_{2}\right) \operatorname{Im} K\left(w_{0}\right)}{r^{2}}- \\
-\left(y_{1}-x_{1}\right) \lambda \int_{0}^{\infty} \frac{\sqrt{u^{2}+\alpha^{2}} \operatorname{Re} K(w)-\left(y_{2}-x_{2}\right) \operatorname{Im} K(w)}{u^{2}+r^{2}} \cdot \frac{I_{1}(\lambda u) d u}{\sqrt{u^{2}+\alpha^{2}}} \\
y \neq x, w_{0}=i\left|y_{1}-x_{1}\right|+y_{2}, I_{1}(\lambda u)=I_{0}^{\prime}(\lambda u)
\end{gathered}
$$

and

$$
\begin{aligned}
& -2 \pi K\left(x_{2}\right) \frac{\partial \Phi(y, x)}{\partial y_{2}}=\frac{\left(y_{2}-x_{2}\right) \operatorname{Re} K\left(w_{0}\right)-\left(y_{1}-x_{1}\right) \operatorname{Im} K\left(w_{0}\right)}{r^{2}}- \\
& -\lambda \int_{0}^{\infty} \frac{\left(y_{2}-x_{2}\right) \operatorname{Re} K(w)-\sqrt{u^{2}+\alpha^{2}} \operatorname{Im} K(w)}{u^{2}+r^{2}} I_{1}(\lambda u) d u, \quad y_{1} \neq x_{1},
\end{aligned}
$$

which are obtained from (2.4). 
Really,

$$
\begin{gathered}
\left|\exp \left[-b i \rho_{1}\left(w-\frac{h}{2}\right)-b_{1} i \rho_{0}\left(w-\frac{h}{2}\right)\right]\right|= \\
=\exp \operatorname{Re}\left[-b i \rho_{1}\left(w-\frac{h}{2}\right)-b_{1} i \rho_{0}\left(w-\frac{h}{2}\right)\right]= \\
=\exp \left[-b \rho_{1} \sqrt{u^{2}+\alpha^{2}} \cos \rho_{1}\left(y_{2}-\frac{h}{2}\right)-b_{1} \rho_{0} \sqrt{u^{2}+\alpha^{2}} \cos \rho_{0}\left(y_{2}-\frac{h}{2}\right)\right] .
\end{gathered}
$$

As

$$
\begin{gathered}
-\frac{\pi}{2} \leq-\frac{\rho_{1}}{\rho} \cdot \frac{\pi}{2} \leq \frac{\rho_{1}}{\rho} \cdot \frac{\pi}{2}<\frac{\pi}{2}, \\
-\frac{\pi}{2} \leq-\frac{\rho_{1}}{\rho} \cdot \frac{\pi}{2} \leq \rho_{0}\left(y_{2}-\frac{h}{2}\right) \leq \frac{\rho_{1}}{\rho} \cdot \frac{\pi}{2}<\frac{\pi}{2} .
\end{gathered}
$$

Consequently,

$$
\cos \rho\left(y_{2}-\frac{h}{2}\right)>0, \cos \rho_{0}\left(y_{2}-\frac{h}{2}\right) \geq \cos \frac{h \rho_{0}}{2}>\delta_{0}>0,
$$

It does not vanish in the region $G$ and

$$
\begin{aligned}
& |\Phi(y, x)|=\mathrm{O}\left[\exp \left(-\varepsilon \rho_{1}\left|y_{1}\right|\right)\right], \varepsilon>0, y \rightarrow \infty, y \in G \bigcup \partial G, \\
& \left|\frac{\partial \Phi(y, x)}{\partial y_{1}}\right|=\mathrm{O}\left[\exp \left(-\varepsilon \rho_{1}\left|y_{1}\right|\right)\right], \varepsilon>0, y \rightarrow \infty, y \in G \bigcup \partial G, \\
& \left|\frac{\partial \Phi(y, x)}{\partial y_{2}}\right|=\mathrm{O}\left[\exp \left(-\varepsilon \rho_{1}\left|y_{1}\right|\right)\right], \varepsilon>0, y \rightarrow \infty, y \in G \bigcup \partial G .
\end{aligned}
$$

We now choose $\rho_{1}$ with the condition $\rho_{2}<\rho_{1}<\rho$. Then condition (2.8) is fulfilled and the integral formula (2.6) is true. Theorem 2.1 is proved.

Condition (2.10) can be weakened.

We denote by $A_{\rho}(G)$ is the class of vector-valued functions from $A(G)$, satisfying the following growth condition:

$$
A_{\rho}(G)=\left\{U(y): U(y) \in A(G),|U(y)| \leq \exp \left[\mathrm{o}\left(\exp \rho\left|y_{1}\right|\right)\right], y \rightarrow \infty, y \in G\right\} .
$$

The following is valid

Theorem 2.2. Suppose $U(y) \in A_{\rho}(G)$ that it satisfies the growth condition

$$
\begin{gathered}
|U(y)| \leq C \exp \left[a \cos \rho_{1}\left(y_{2}-\frac{h}{2}\right) \exp \left(\rho_{1}\left|y_{1}\right|\right)\right], \\
a \geq 0,0<\rho_{1}<\rho, y \in \partial G,
\end{gathered}
$$

where $C-$ is some constant. Then formula (2.6) is valid.

Proof. Divide the area $G$ by a line $y_{2}=\frac{h}{2}$ into two areas

$$
G_{1}=\left\{y: 0<y_{2}<\frac{h}{2}\right\} \text { and } G_{2}=\left\{y: \frac{h}{2}<y_{2}<h\right\} \text {. }
$$


Consider the domain $G_{1}$. In the formula (2.4) together $K(w)$ we put $K_{1}(w)$

$$
\begin{gathered}
K_{1}(w)=K(w) \exp \left[-\delta i \tau\left(w-\frac{h}{2}\right)-\delta_{1} i \rho\left(w-\frac{h}{2}\right)\right], \\
\rho<\tau<2 \rho, \delta>0, \delta_{1}>o,
\end{gathered}
$$

Here $K(w)$ it is determined from (2.10). With this notation, (2.8) is true.

Really,

$$
\begin{aligned}
& \left|\exp \left[-i \tau\left(w-\frac{h}{4}\right)-\delta_{1} i \rho\left(w-\frac{h}{4}\right)\right]\right|= \\
= & \exp \left[-\delta \tau \sqrt{u^{2}+\alpha^{2}} \cos \tau\left(y_{2}-\frac{h}{4}\right)\right]= \\
= & \exp \left[-\delta \tau \sqrt{u^{2}+\alpha^{2}}\right] \leq \exp \left[-\delta \exp \tau\left|y_{1}\right|\right],
\end{aligned}
$$

as

$-\frac{\pi}{2} \leq-\tau \frac{\pi}{4} \leq \tau\left(y_{2}-\frac{h}{4}\right) \leq \tau \frac{\pi}{2}<\frac{h}{2}$ and $\cos \tau\left(y_{2}-\frac{h}{4}\right) \geq \cos \tau \frac{h}{4} \geq \delta_{0}>0$.

We denote the corresponding $\Phi(y, x)$ by $\Phi^{+}(y, x)$. As

$$
\cos \tau\left(y_{2}-\frac{h}{4}\right) \geq \delta_{0}, y \in G_{1} \bigcup \partial G_{1},
$$

then for fixed $x \in G_{1}, y \in G_{1} \bigcup \partial G_{1}$, for $\Phi^{+}(y, x)$ and its derivatives are true asymptotic estimates

$$
\begin{aligned}
& \left|\Phi^{+}(y, x)\right|=\mathrm{O}\left[\exp \left(-\delta_{0} \exp \left(\tau\left|y_{1}\right|\right)\right], y \rightarrow \infty, \rho<\tau<2 \rho,\right. \\
& \left|\frac{\partial \Phi^{+}(y, x)}{\partial y_{1}}\right|=\mathrm{O}\left[\exp \left(-\delta_{0} \exp \left(\tau\left|y_{1}\right|\right)\right], y \rightarrow \infty, \rho<\tau<2 \rho,\right. \\
& \left|\frac{\partial \Phi^{+}(y, x)}{\partial y_{2}}\right|=\mathrm{O}\left[\exp \left(-\delta_{0} \exp \left(\tau\left|y_{1}\right|\right)\right], y \rightarrow \infty, \rho<\tau<2 \rho .\right.
\end{aligned}
$$

Suppose $U(y) \in A\left(G_{1}\right)$ that in a domain $G_{1}$ satisfies the growth condition

$$
|U(y)| \leq C \exp \left[\exp (2 \rho-\varepsilon)\left|y_{1}\right|\right], \varepsilon>0 .
$$

We choose $\tau$ the inequality $2 \rho-\varepsilon<\tau<2 \rho$ in (2.15).

Then the condition (2.15) is satisfied for the region $G_{1}$, therefore, the following integral formula holds

$$
U(x)=\int_{\partial G_{1}} M(y, x) U(y) d s_{y}, x \in G_{1} .
$$

where

$$
M(y, x)=\left(E\left(\Phi^{+}(y, x) u^{0}\right) D^{*}\left(\frac{\partial}{\partial x}\right)\right) D\left(t^{T}\right) .
$$

If $U(y) \in A\left(G_{2}\right)$ satisfies the growth condition (2.14) in $G_{2}$, then for $2 \rho-\varepsilon<$ $\tau<2 \rho$, similarly we obtain the following integral formula

$$
U(x)=\int_{\partial G_{2}} M(y, x) U(y) d s_{y}, x \in G_{2} .
$$


where

$$
M(y, x)=\left(E\left(\Phi^{-}(y, x) u^{0}\right) D^{*}\left(\frac{\partial}{\partial x}\right)\right) D\left(t^{T}\right) .
$$

Here $\Phi^{-}(y, x)$ it is defined by the formula (2.4), in which $K(w)$ it is replaced by the function $K_{2}(w)$ :

$$
K_{2}(w)=K(w) \exp \left[-\delta i \tau\left(w-h_{1}\right)-\delta_{1} i \rho\left(w-\frac{h}{2}\right)\right],
$$

where

$$
h_{1}=\frac{h}{2}+\frac{h}{4}, \frac{h}{2}<y_{2}<h, \frac{h}{2}<x_{2}<h_{1}, \delta>0, \delta_{1}>0 .
$$

In the formulas obtained with this formula, the integrals (according to (2.9)) converge uniformly for $\delta \geq 0$, when $U(y) \in A_{\rho}(G)$. In these formulas we put $\delta=0$ and, combining the formulas obtained, we find

$$
U(x)=\int_{\partial G} M(y, x) U(y) d s_{y}, \quad x \in G, x_{2} \neq \frac{h}{2},
$$

where

$$
M(y, x)=\left(E\left(\tilde{\Phi}(y, x) u^{0}\right) D^{*}\left(\frac{\partial}{\partial y}\right)\right) D\left(t^{T}\right) .
$$

(integrals over the cross section $y_{2}=\frac{h}{2}$ are mutually destroyed)

$$
\tilde{\Phi}(y, x)=\left(\Phi^{+}(y, x)\right)_{\delta=0}=\left(\Phi^{-}(y, x)\right)_{\delta=0} .
$$

Here, $\tilde{\Phi}(y, x)$ is determined by the formula $(2.4)$, in which $K(w)$ is determined from (2.15), where $\delta=0$ is laid. According to the continuation principle, formula (2.20) is true for $\forall x \in G$. Under condition (2.16), formula (2.20) is true for $\forall \delta_{1} \geq 0$. Assuming $\delta_{1}=0$, we obtain the proof of the theorem. Theorem 2.2 is proved.

In the formula (2.4), choosing

$$
\begin{gathered}
K(w)=\frac{1}{w-x_{2}+3 h} \exp (\sigma w), \\
K\left(x_{2}\right)=\frac{1}{3 h} \exp \left(\sigma x_{2}\right), 0<x_{2}<h, h=\frac{\pi}{\rho},
\end{gathered}
$$

we get

$$
\Phi_{\sigma}(y, x)=-\frac{e^{-\sigma x_{2}}}{2 \pi(3 h)^{-1}} \int_{0}^{\infty} \operatorname{Im} \frac{\exp (\sigma w)}{\left(w-x_{2}+3 h\right)\left(w-x_{2}\right)} \frac{u I_{0}(\lambda u)}{\sqrt{u^{2}+\alpha^{2}}} d u .
$$

Then the integral formula (2.6) has the following form:

$$
U(x)=\int_{\partial G} N_{\sigma}(y, x) U(y) d s_{y}, \quad x \in G,
$$

where

$$
N_{\sigma}(y, x)=\left(E\left(\Phi_{\sigma}(y, x) u^{0}\right) D^{*}\left(\frac{\partial}{\partial y}\right)\right) D\left(t^{T}\right)
$$


Suppose that the boundary of the domain $G$ consists of a hyper plane $y_{2}=0$ and a smooth curve $S$ extending to infinity and lying in the strip

$$
0<y_{2}<h, h=\frac{\pi}{\rho}, \rho>0 .
$$

We assume that $S$ is given by the equation

$$
y_{2}=\psi\left(y_{1}\right),-\infty<y_{1}<\infty,
$$

where $\psi\left(y_{1}\right)$ satisfies the condition

$$
\left|\psi^{\prime}\left(y_{1}\right)\right| \leq M<\infty, M=\text { const. }
$$

\section{Regularization of the Cauchy problem}

Formulation of the problem. Suppose that $U(y) \in A_{\rho}(G)$ and

$$
\left.U(y)\right|_{S}=f(y), y \in S .
$$

Here, $f(y)$ - a given continuous vector-valued function on $S$.

It is required to restore the vector function $U(y)$ in the region $G$, based on its values $f(y)$ on $S$.

The following is valid

Theorem 3.1. Let $U(y) \in A_{\rho}(G)$ it satisfy the inequality

$$
|U(y)| \leq 1, y \in T \text {. }
$$

If

$$
U_{\sigma}(x)=\int_{S} N_{\sigma}(y, x) U(y) d s_{y}, \quad x \in G
$$

then the following estimate holds

$$
\left|U(x)-U_{\sigma}(x)\right| \leq C_{\rho}(\lambda, x) \sigma e^{-\sigma x_{2}}, \sigma>1, x \in G .
$$

Here and below functions bounded on compact subsets of the domain $G$, we denote by $C_{\rho}(\lambda, x)$.

Proof. Using the integral formula (2.23) and the equality (3.3), we obtain

$$
U(x)=U_{\sigma}(x)+\int_{T} N_{\sigma}(y, x) U(y) d s_{y}, x \in G .
$$

Taking inequality (3.2) into account, we estimate the following

$$
\left|U(x)-U_{\sigma}(x)\right| \leq \int_{T}|U(y)|\left|N_{\sigma}(y, x)\right| d s_{y} \leq \int_{T}\left|N_{\sigma}(y, x)\right| d s_{y}, x \in G .
$$

To do this, we estimate the integrals $\int_{y_{2}=0}\left|\Phi_{\sigma}(y, x)\right| d s_{y}, \int_{y_{2}=0}\left|\frac{\partial \Phi_{\sigma}(y, x)}{\partial y_{1}}\right| d s_{y}$ and $\int_{y_{2}=0}\left|\frac{\partial \Phi_{\sigma}(y, x)}{\partial y_{2}}\right| d s_{y}$ on the part $T$ of the plane $y_{2}=0$. 
Let $\sigma>0$. Separating the imaginary part of (2.22), we obtain

$$
\begin{aligned}
& \Phi_{\sigma}(y, x)=\frac{e^{\sigma\left(y_{2}-x_{2}\right)}}{2 \pi(3 h)^{-1}}\left[\int _ { 0 } ^ { \infty } \left(\frac{\left(\beta+\beta_{1}\right) \cos \sigma \alpha_{1}}{\left(\alpha_{1}^{2}+\beta_{1}^{2}\right)\left(\alpha_{1}^{2}+\beta^{2}\right)}+\right.\right. \\
& \left.\left.+\frac{\left(-\alpha_{1}^{2}+\beta_{1} \beta\right)}{\left(\alpha_{1}^{2}+\beta_{1}^{2}\right)\left(\alpha_{1}^{2}+\beta^{2}\right)} \frac{\sin \sigma \alpha_{1}}{\alpha_{1}}\right) u I_{0}(\lambda u) d u\right],
\end{aligned}
$$

where

$$
\alpha_{1}^{2}=u^{2}+\alpha^{2}, \beta=y_{2}-x_{2}, \beta_{1}=y_{2}-x_{2}+3 h
$$

We estimate first $\int_{y_{2}=0}\left|\Phi_{\sigma}(y, x)\right| d s_{y}$. Taking into account equality (3.5) and inequality

$$
I_{0}(\lambda u) \leq \exp (\lambda u)
$$

we have

$$
\int_{y_{2}=0}\left|\Phi_{\sigma}(y, x)\right| d s_{y} \leq C_{\rho}(\lambda, x) \sigma e^{-\sigma x_{2}}, \sigma>1, x \in G .
$$

To estimate the integrals $\int_{y_{2}=0}\left|\frac{\partial \Phi_{\sigma}(y, x)}{\partial y_{1}}\right| d s_{y}$ and $\int_{y_{2}=0}\left|\frac{\partial \Phi_{\sigma}(y, x)}{\partial y_{2}}\right| d s_{y}$, we use equalities (2.11) and (2.12). For this, using equalities (2.21) and choosing

$$
K\left(w_{0}\right)=\exp \left(\sigma w_{0}\right), \sigma>0,
$$

we obtain the following formulas

$$
\begin{gathered}
-\frac{2 \pi e^{\sigma x_{2}}}{(3 h)^{-1}} \frac{\partial \Phi_{\sigma}}{\partial y_{1}}=\frac{\left(y_{1}-x_{1}\right) \operatorname{Re} \exp \left(\sigma w_{0}\right)+\operatorname{sign}\left(y_{1}-x_{1}\right)\left(y_{2}-x_{2}\right) \operatorname{Im} \exp \left(\sigma w_{0}\right)}{r^{2}}- \\
-\left(y_{1}-x_{1}\right) \lambda \int_{0}^{\infty} \frac{\sqrt{u^{2}+\alpha^{2}} \operatorname{Re} \exp (\sigma w)-\left(y_{2}-x_{2}\right) \operatorname{Im} \exp (\sigma w)}{u^{2}+r^{2}} \cdot \frac{I_{1}(\lambda u) d u}{\sqrt{u^{2}+\alpha^{2}}}, y \neq x,
\end{gathered}
$$

and

$$
\begin{gathered}
-\frac{2 \pi e^{\sigma x_{2}}}{(3 h)^{-1}} \frac{\partial \Phi_{\sigma}}{\partial y_{2}}=\frac{\left(y_{2}-x_{2}\right) \operatorname{Re} \exp \left(\sigma w_{0}\right)+\left(y_{1}-x_{1}\right) \operatorname{Im} \exp \left(\sigma w_{0}\right)}{r^{2}}- \\
-\lambda \int_{0}^{\infty} \frac{\left(y_{2}-x_{2}\right) \operatorname{Re} \exp (\sigma w)-\sqrt{u^{2}+\alpha^{2}} \operatorname{Im} \exp (\sigma w)}{u^{2}+r^{2}} I_{1}(\lambda u) d u, \quad y_{1} \neq x_{1} .
\end{gathered}
$$

Taking into account equality (3.9) and inequality

$$
I_{1}(\lambda u) \leq \lambda u \exp (\lambda u)
$$

we get

$$
\int_{y_{2}=0}\left|\frac{\partial \Phi_{\sigma}(y, x)}{\partial y_{1}}\right| d s_{y} \leq C_{\rho}(\lambda, x) \sigma e^{-\sigma x_{2}}, \sigma>1, x \in G
$$


Analogously, taking into account equality (3.10) and inequality $(3,12)$, we estimate the following integral

$$
\int_{y_{2}=0}\left|\frac{\partial \Phi_{\sigma}(y, x)}{\partial y_{2}}\right| d s_{y} \leq C_{\rho}(\lambda, x) \sigma e^{-\sigma x_{2}}, \sigma>1, x \in G .
$$

From the inequalities (3.7), (3.12), and (3.13), we obtain (3.4). Theorem 3.1 is proved.

Corollary 3.1. The limiting equality

$$
\lim _{\sigma \rightarrow \infty} U_{\sigma}(x)=U(x)
$$

holds uniformly on each compact set in the domain $G$.

Theorem 3.2. Let $U(y) \in A_{\rho}(G)$ satisfy condition (3.2) on a part of the plane $y_{2}=0$, and on a smooth curve $S$ the inequality

$$
|U(y)| \leq \delta, \quad 0<\delta<1 .
$$

Then the following estimate holds

$$
|U(x)| \leq C_{\rho}(\lambda, x) \sigma \delta^{\frac{x_{2}}{h}}, \sigma>1, x \in G .
$$

Proof. Using the integral formula (2.23), we have

$$
\begin{gathered}
U(x)=\int_{\partial G} N_{\sigma}(y, x) U(y) d s_{y}= \\
=\int_{S} N_{\sigma}(y, x) U(y) d s_{y}+\int_{T} N_{\sigma}(y, x) U(y) d s_{y}, x \in G .
\end{gathered}
$$

Taking into account the boundary condition (3.2) and inequality (3.14), we obtain the estimate

$$
\begin{aligned}
|U(x)| & \leq \int_{S}|U(y)|\left|N_{\sigma}(y, x)\right| d s_{y}+\int_{T}|U(y)|\left|N_{\sigma}(y, x)\right| d s_{y} \leq \\
& \leq \delta \int_{S}\left|N_{\sigma}(y, x)\right| d s_{y}+\int_{T}\left|N_{\sigma}(y, x)\right| d s_{y}, x \in G .
\end{aligned}
$$

First we estimate the first integral of inequality (3.16). To do this, we estimate the integrals $\int_{S}\left|\Phi_{\sigma}(y, x)\right| d s_{y}, \int_{S}\left|\frac{\partial \Phi_{\sigma}(y, x)}{\partial y_{1}}\right| d s_{y}$ and $\int_{S}\left|\frac{\partial \Phi_{\sigma}(y, x)}{\partial y_{2}}\right| d s_{y}$ on a smooth curve $S$.

Taking into account equality (3.5) and inequality (3.6), we have

$$
\int_{S}\left|\Phi_{\sigma}(y, x)\right| d s_{y} \leq C_{\rho}(\lambda, x) \sigma e^{\sigma\left(h-x_{2}\right)}, \sigma>1, x \in G .
$$

Using (3.9) and inequality (3.11), we have

$$
\int_{S}\left|\frac{\partial \Phi_{\sigma}(y, x)}{\partial y_{1}}\right| d s_{y} \leq C_{\rho}(\lambda, x) \sigma e^{\sigma\left(h-x_{2}\right)}, \sigma>1, x \in G .
$$


Similarly using (3.10) and inequality (3.11), we obtain

$$
\int_{S}\left|\frac{\partial \Phi_{\sigma}(y, x)}{\partial y_{2}}\right| d s_{y} \leq C_{\rho}(\lambda, x) \sigma e^{\sigma\left(h-x_{2}\right)}, \sigma>1, x \in G .
$$

From (3.17) - (3.19), we obtain

$$
\left|\int_{S} N_{\sigma}(y, x) U(y) d s_{y}\right| \leq C_{\rho}(\lambda, x) \sigma \delta e^{\sigma\left(h-x_{2}\right)}, \sigma>1, x \in G .
$$

The following is known

$$
\left|\int_{T} N_{\sigma}(y, x) U(y) d s_{y}\right| \leq C_{\rho}(\lambda, x) \sigma e^{-\sigma x_{2}}, \sigma>1, x \in G .
$$

Now taking into account (3.16), (3.20) - (3.21), we have

$$
|U(x)| \leq \frac{C_{\rho}(\lambda, x) \sigma}{2}\left(\delta e^{\sigma h}+1\right) e^{-\sigma x_{2}}, \sigma>1, x \in G .
$$

Choosing $\sigma$ from equality

$$
\sigma=\frac{1}{h} \ln \frac{1}{\delta},
$$

we obtain the inequality (3.15). Theorem 3.2 is proved.

Let $U(y) \in A_{\rho}(G)$ and together with $U(y)$ on $S$ it is given its approximation $f_{\delta}(y)$, respectively, with an error $0<\delta<1, \max _{S}\left|U(y)-f_{\delta}(y)\right| \leq \delta$.

We set

$$
U_{\sigma(\delta)}(x)=\int_{S} N_{\sigma}(y, x) f_{\delta}(y) d s_{y}, x \in G .
$$

The following is valid

Theorem 3.3. Let $U(y) \in A_{\rho}(G)$ it satisfy condition (3.2) on a part of the plane $y_{2}=0$.

Then the following estimate holds

$$
\left|U(x)-U_{\sigma(\delta)}(x)\right| \leq C_{\rho}(\lambda, x) \sigma \delta^{\frac{x_{2}}{h}}, \sigma>1, x \in G .
$$

Proof. From the integral formulas (2.23) and (3.23), we have

$$
U(x)-U_{\sigma(\delta)}(x)=\int_{S} N_{\sigma}(y, x)\left\{U(y)-f_{\delta}(y)\right\} d s_{y}+\int_{T} N_{\sigma}(y, x) U(y) d s_{y} .
$$

Now, repeating the proof of Theorems 3.1 and 3.2, we obtain

$$
\left|U(x)-U_{\sigma(\delta)}(x)\right| \leq \frac{C(x) \sigma}{2}\left(\delta e^{\sigma h}+1\right) e^{-\sigma x_{2}} .
$$

Hence, choosing $\sigma$ from (3.22), we obtain (3.24).

Corollary 3.2. The limiting equality

$$
\lim _{\delta \rightarrow 0} U_{\sigma(\delta)}(x)=U(x),
$$

holds uniformly on each compact set in the domain $G$.

Thus, the functional $\left.U_{\sigma(\delta}\right)(x)$ determines the regularization of the solution of problem (2.23), (3.23). 
Acknowledgments. In conclusion, the author expresses his sincere gratitude to the professor of Potsdam University, the Institute of Mathematics, Doctor of Physical and Mathematical Sciences, Professor, N.N. Tarkhanov, to the professor Institute of Mathematics and Fundamental Informatics, Siberian Federal University, Doctor of Physical and Mathematical Sciences, Professor, A.A. Shlapunov and to the academician of the Republic of Uzbekistan, Doctor of Physical and Mathematical Sciences, Academician, Sh.A. Alimov for useful advice.

\section{REFERENCES}

[1] N.N. Tarkhanov, On the Carleman matrix for elliptic systems, Dokl. Akad. Nauk SSSR. 284 (1985), no. 2., 294-297. (in Russian).

[2] N.N. Tarkhanov, Tarkhanov, Solvability criterion for the ill-posed Cauchy problem for elliptic systems, Dokl. Akad. Nauk SSSR. 380 (1989), no. 3., 531-534. (in Russian).

[3] N.N. Tarkhanov, The Cauchy problem for solutions of elliptic equations, Akad. Verl., Berlin, V. 7. 1995

[4] T. Carleman, Les fonctions quasi analytiques, Gautier-Villars et Cie., Paris, 1926.

[5] M.M. Lavrent'ev, On the Cauchy problem for second-order linear elliptic equations, Dokl. Akad. Nauk SSSR. 112 (1957), no. 2., 195-197.

[6] M.M. Lavrent'ev, On some ill-posed problems of mathematical physics, Nauka, Novosibirsk, 1962 (in Russian).

[7] Sh. Yarmukhamedov, On the Cauchy problem for the Laplace equation, Dokl. Akad. Nauk SSSR. 235 (1977), no. 2., 281-283. (in Russian).

[8] Sh. Yarmukhamedov, On the extension of the solution of the Helmholtz equation, Dokl. Ross. Akad. Nauk. 357 (1997), no. 3., 320-323. (in Russian).

[9] Sh. Yarmukhamedov, The Carleman function and the Cauchy problem for the Laplace equation, Sibirsk. Math. Journal. 45 (2004), no. 3., 702-719. (in Russian).

[10] J. Adamar, The Cauchy problem for linear partial differential equations of hyperbolic type, Nauka, Moscow, 1978 (in Russian).

[11] L.A. Aizenberg, Carleman's formulas in complex analysis, Nauka, Novosibirsk, 1990 (in Russian).

[12] G.M. Goluzin, V.M. Krylov, The generalized Carleman formula and its application to the analytic continuation of functions, Math. Sbornik. 40 (1993), no. 2., 144-149. (in Russian).

[13] A.N. Tikhonov, On the solution of ill-posed problems and the regularization method, Dokl. Akad. Nauk SSSR. 151 (1963), no. 3., 501-504. (in Russian).

[14] A. Bers, F. John, M. Shekhter Partial Differential Equations, Mir, Moscow, 1966 (in Russian).

[15] M.A. Aleksidze, Fundamental functions in approximate solutions of boundary value problems, Nauka, Moscow, 1991 (in Russian).

[16] E.V. Arbuzov, A.L. Bukhgeim, The Carleman formula for the Helmholtz equation, Sib. Math. Journal. 47 (1979), no. 3., 518-526. (in Russian).

[17] A.A. Shlapunov, On the Cauchy problem for the Laplace equation, Siberian Mathematical Journal. 43 (1992), no. 4., 953-963.

[18] A.A. Shlapunov, Boundary problems for Helmholtz equation and the Cauchy problem for Dirac operators, J. Sib. Fed. Univ. Math. Phys., 4 (2011), no. 2., 217-228.

[19] I.E. Niyozov, O.I. Makhmudov The Cauchy problem of the moment elasticity theory in $\mathbb{R}^{m}$, Izv. Vuz. Mat. 58 (2014), no. 2., 24-30.

[20] D.A. Juraev, The construction of the fundamental solution of the Helmholtz equation, Reports of the Academy of Sciences of the Republic of Uzbekistan. (2012), no. 2., 14-17. (in Russian).

[21] D.A. Juraev, The Cauchy problem for matrix factorizations of the Helmholtz equation in an unbounded domain, Sib. Electron. Mat. Izv. 14 (2017), 752-764. (in Russian). doi:10.17377/semi.2017.14.064, Zbl 1375.35153.

[22] D.A. Zhuraev, Cauchy problem for matrix factorizations of the Helmholtz equation, Ukrainian Mathematical Journal. 69 (2018), no. 10., 1583-1592. (Ukrainian Original Vol. 69, No. 10, October, 2017). (in Russian).

doi:10.1007/s11253-018-1456-5. 
[23] D.A. Juraev, On the Cauchy problem for matrix factorizations of the Helmholtz equation in a bounded domain, Sib. Electron. Mat. Izv. 15 (2018), 11-20. (in Russian). doi:10.17377/semi.2018.15.002, Zbl 1387.35176.

[24] D.A. Juraev, On the Cauchy problem for matrix factorizations of the Helmholtz equation in a bounded domain, Journal of Universal Mathematics. 1 (2018), no. 3, 312-319.

[25] D.A. Juraev, On the Cauchy problem for matrix factorizations of the Helmholtz equation in an unbounded domain in $\mathbb{R}^{2}$, Sib. Electron. Mat. Izv. 15 (2018), 1865-1877. (in Russian). doi.org/10.33048/semi.2018.15.151.

[26] D.A. Juraev, On a regularized solution of the Cauchy problem for matrix factorizations of the Helmholtz equation, SAdvanced Mathematical Models \& Applications 4 (2019), no. 1, 86-96.

KARSHI STATE UNIVERSITY, PHYSICS-MATHEMATICS, 180100, KARSHI, UZBEKISTAN

Email address: juraev_davron@list.ru 